\title{
A model for optimal economic lockdown to contain epidemic
}

\author{
Marco Tognoli ( $\square$ tognaz72@gmail.com ) \\ https://orcid.org/0000-0003-2120-390X
}

\section{Case Report}

Keywords: Boolean linear programming, input-output model, economic lockdown, covid-19 pandemic

Posted Date: February 7th, 2022

DOI: https://doi.org/10.21203/rs.3.rs-1335145/v1

License: (9) This work is licensed under a Creative Commons Attribution 4.0 International License. Read Full License 


\title{
A model for optimal economic lockdown to contain epidemic
}

\author{
Marco Tognoli
}

\begin{abstract}
This paper is concerned with the application of operations research in defining the optimal lockdown of economic activities to contain epidemic. The problem of optimal lockdown consists in deciding as best economic sectors can be lockdown with respect to fundamental sectors (essential goods and services) while disruptive impacts are minimized on the economy as a whole. Many countries around the world are currently implementing the lockdown of most economic activities to contain the spread of the Covid-19 pandemic. The lockdown brings health benefits for the society as it contains the spread of the virus, reducing the number of infections and allowing the health system to treat those infected better. This paper describes a Boolean linear programming model to deal with the problem of selecting several economic sectors to be shutdown. The objective function is linear and the constraints are linear inequalities related to the Leontief's input-output table. The model permits to analyze the feasibility of national economic system in which some elements of the input-output table are set equal to zero. The mathematical approach to the shutdown problem permits to identify the greatest number of economic sectors that can be closed without destroying the fundamental sectors. Since solution of the shutdown problem is the greatest number of lockdown economic sectors, author believes the model allows to oppose effectively the spread of virus. Once spread of the virus decreases, another feature of the model is to support decision makers in assigning priorities to the economic sectors to be gradually unlocked.
\end{abstract}

Standard input-output models are able to reveal how different sectors of an economy are interconnected and how changes in one sector affect all other sectors. Besides the use of the input-output data for descriptive analyses of economic interrelations, this table also provides the empirical fundament for a wide scale of impact analysis. inputoutput models greatly differ in size and possible applications. Simple static input-output models are used for comparative-static impact (scenario) analysis. With the help of input-output quantity models, statements can be made about direct and indirect effects based on exogenous changes in demand. More complex dynamic input-output models 
largely resolve the limitations and inherent assumptions of static input-output models. Time is considered explicitly; quantity and price reactions are modelled endogenously in a holistic approach and feedback effects are captured. Last models are not only suitable for scenario analysis and for forecasting as well. In "A Linear Programming Solution to Dynamic Leontief type Models" Harvey M. Wagner [1] presents a general dynamic model of an economy and investigates a number of questions related to the feasibility of certain time profiles of demand and the rate of substitution between economic activities. The Inoperability Input-Output Model [2], [3]. [4] was developed to understand better the infrastructure interdependencies. Based on the Leontief InputOutput Model, the model is demand-driven, wherein perturbations to the final demand levels are considered the initiating event, and the impact to sectors' production outputs are the direct and indirect effects resulting from sector interdependencies. Case study shows that the different perturbation classes (demand or supply, quantity or price) yield different rank orders of sectors impacted by the initiating event, thus providing various perspectives of impacts.

The present study differs from most previous studies in two aspects. First, linear programming techniques have been widely used in Input-Output model: from the primary and dual formulation of the problem to the analysis of Lagrange multipliers in order to assess the mutual relative importance of sectors. Second, the impact of lockdown is measured in terms of annihilated interdependencies rather than in terms of canceled economic values such as revenues and monetary exchanges. For this reason, the proposed model takes into account the adjacency matrix got from input-output Table rather than the Table itself. This analysis is static in character (it is a static I-O model) because it does not take care explicitly of time, quantity and price reactions, consumption and production lags, growth of final demand (these ones are known as dynamic I-O models). The study simply considers the interruption of several activity as a consequence of a government measure adopted to reduce the risk of contagion. The proposed model answers to the basic question if the annihilation of $\mathrm{N}$ sectors is feasible, secondary the model identifies in the Lagrange multipliers the means to sequencing the sectors to be reopened.

Keywords: Boolean linear programming; economic planning; input-output model; economic lockdown; covid-19 pandemic 
1. Introduction: Never like before today, we have become aware we all live in a globalized, complex and interconnected world. A microscopic virus from the parts of Wuhan is enough to trigger panic into financial markets, block international flights, slow the global economy, mobilize governments in a maximum reduction of the risk of contagion. There are numerous papers on the economic costs of epidemics: for example, "Economic Cost of Dengue in Puerto Rico" look at the medical costs of dengue in Puerto Rico of 2010; Armien, Blas, Jose A. Suaya, Evelia Quiroz, Binod K. Sah, Vicente Bayard, Loyd Marchena, Cornelio Campos, and Donald S. Shepard do a study for Panama in "Clinical characteristics and National Economic Cost of the 2005 Dengue Epidemic in Panama.". The recent study of Shaowen Luo, Kwok Ping Tsang in "China and World Output Impact of the Hubei Lockdown During the Coronavirus Outbreak" (2020) [5] provides an estimate on the economic impact of the coronavirus on China and the global economy. Using a network approach, they estimate the output loss due to the lockdown of the Hubei province triggered by the coronavirus disease.

During the first part of twentieth century the economist and mathematician Wassily W.Leontief [6], [7], [8] studied the interactions existing among national industrial sectors. The core of the analysis was made up input-output table. Input-output table is a double entry table which summarizes all commercial exchanges occurred inside an assigned economy for a certain period of time. In brief, input-output table gives a schematic and abstract portrait of relations emerging from production and flow of goods and services between economic sectors. Secondary, input-output table permits to develop several economic models such as for example simulating how the lockdown of an economic sector influences the whole economy. The present paper starts from the input-output table and it analyzes if it is feasible to nullify some element of input-output table or, in other words, if it is possible to lockdown the maximum number of sectors while a certain type of sectors must remain open.

On 22nd march the "Close Italy" Decree no.18/2020 ("Chiudi Italia") have been issued and it identified the essential activities that must remain open, while the other deemed unnecessary (non-essential) must stop all activities throughout the whole italian territory. In order to keep Italy's supply chain running essential sectors were listed in an annex enclosed in the Decree. If "Close Italy" Decree is aimed to oppose the spread of contagions within the Italian population, the objective of the mathematical model is to 
analyze how many and which economic sectors can be closed without generating tensions inside the Italian economic system.

The interdependence of different economic sectors has been represented by an oriented graph in which each node (vertex) represents a sector, the edge from vertex $\mathrm{i}$ to vertex $\mathrm{j}$ represent the goods produced by sector $\mathrm{i}$ and sold to sector $\mathrm{j}$, the incident edges on $\mathrm{i}$-th vertex represent the goods/services purchased by $\mathrm{i}$-th sector.

An input-output table with $\mathrm{n}$ rows and $\mathrm{n}$ columns is therefore transformed into an adjacency matrix, nxn. Each sector is associated with a Boolean variable being the unknown element of the problem. For nxn adjacency matrix the number of variables is equal to $\mathrm{n}$. If the variable takes value 1 it means that the sector is essential and cannot be closed, while if the variable takes value 0 it means that the sector is not essential and therefore can be closed. Main goal of the model is to minimize the sum of all Boolean variables (objective function). The variables of the problem are bound to assume a certain number of unit values corresponding to the $\mathrm{N}$ sectors deemed as strategic by the legislator so that the supply of these $\mathrm{N}$ sectors can be guaranteed. The feasible region consists of $2 \mathrm{n}$ constraints and it is intimately connected to the adjacency matrix. The first type of n constraints ensures that at least $\mathrm{N}$ strategic sectors remain open, while the remaining $\mathrm{n}$ constraints determine which sectors are directly or indirectly involved in supplying the strategic sectors.

The paper analyzes the "Close Italy" Decree in which 47 sectors are defined as strategic. The mathematical model identifies how many and what economic sectors can be closed without interfering with the 47 sectors indicated by the Decree as strategic. The adopted input-output table and its adjacency matrix are based on Supply and Use Table which is made available by the Istituto nazionale di statistica italiano, shortly Istat.

Istat provides two different kind of Supply and Use Tables: the first one refers to 20 sectors and 20 product grouping, while the second one refers to 63 sectors and 63 product grouping. This paper has chosen the level of details based on 63 economic sectors and 63 product groups for Supply table [10]. Rows represent the outputs (sales of groupings of products), while the columns represent the inputs (purchasing of 63 sectors of economic activities). The generic element of the $\mathrm{q}_{\mathrm{ij}}$ table is the economic value of goods / services produced by the $\mathrm{i}$-th sector and sold to the $\mathrm{j}$-th sector as a supply. The economic value of the goods is valorized at the base price. The Istat Supply 
table used for analysis is 2016 "supply_63b.xls" which refers to 2016 and it was published in February 2020. Since the Supply table describes the formation of the supply of goods and services, both domestic and imported production for the year 2016: in this analysis, imports have been excluded.

Once the adjacency matrix $63 \times 63$ has been got, the software used to solve the linear programming problem was "OpenSolver" that works in Microsoft Excel. 
2. General formulation: The interdependencies between different sectors of a national economy may be pictured by means of Leontief's input-output table [6] whose rows stand for an ensemble of products and columns corresponds to economic sectors.

Consider a national economy composed of $\mathrm{n}$ sectors $\mathrm{P}_{1}, \mathrm{P}_{2}, \ldots, \mathrm{P}_{\mathrm{n}}$ that have to satisfy $\mathrm{n}$ final demands of supplies $\mathrm{s}_{1}, \mathrm{~s}_{2}, \ldots, \mathrm{s}_{\mathrm{n}}$. If Legislator established a priori that the $\mathrm{i}$-th demand $\mathrm{s}_{\mathrm{i}}$ is strategic then assign $1 \mathrm{~s}_{\mathrm{i}}=1$, otherwise assign $\mathrm{s}_{\mathrm{i}}=0$ meaning that sector $P_{i}$ should be closed.

Let $A$ the adjacency square matrix related to input-output table. The adjacency matrix consists of a binary matrix whose rows stand for an ensemble of products and columns corresponds to economic sectors: every element of the adjacency matrix contains either 0 or 1 . At the generic position $(i, j)$ there is 1 if and only if a transfer of goods / services from the i-th ensemble to the $\mathrm{j}$-th sector exists otherwise the value is equal to 0 .

$$
A=\left(\begin{array}{lll}
\vec{a}^{1} & \ldots & \vec{a}^{n}
\end{array}\right)=\left(\begin{array}{ccc}
a_{11} & \ldots & a_{1 n} \\
\vdots & \ddots & \vdots \\
a_{n 1} & \ldots & a_{n n}
\end{array}\right)=\left(\begin{array}{c}
\vec{a}_{1} \\
\vdots \\
\vec{a}_{n}
\end{array}\right) \text { where } \mathrm{a}_{\mathrm{ij}} \in\{0 ; 1\}
$$

The economy must produce a well-defined type of goods / services considered strategic for the survival of the Country, but at the same time it is worth to understand if a so restricted economy could run. The proposed linear program model aims to determines which sectors can be closed without compromising the execution of activities within the strategic sectors.

The unknown variables of the problem are defined as Boolean-valued variables: $\mathrm{x}_{\mathrm{i}} \in\{0 ; 1\}$ where $\mathrm{i}=1, \ldots, \mathrm{n}$. Every Boolean variable is associated uniquely with each sector:

- $\mathrm{x}_{\mathrm{i}}=1$ if $\mathrm{i}$-th sector is essential

- $\mathrm{x}_{\mathrm{i}}=0$ if $\mathrm{i}$-th sector is not essential and therefore can be lockdown

The variable of the problem is a row vector of $\mathrm{n}$ coordinates

$$
\vec{x}=\left(x_{1}, x_{2}, \ldots, x_{n}\right)
$$

The parameters of the problem are the final demand of supply for strategic goods and services. Parameters are assigned as a column vector.

$$
\vec{s}=\left(\begin{array}{c}
s_{1} \\
\vdots \\
s_{n}
\end{array}\right) \text { where } \mathrm{s}_{\mathrm{i}} \in\{0 ; 1\}
$$

Legislator declares to keep opened $N=\sum_{i=1}^{n} s_{i}$ sectors. 
Let $\Phi(\vec{x})=M=\sum_{i=1}^{n} x_{i}$ be the function counting the number of opened sectors, $\Phi:[0,1]^{n} \rightarrow[1,2, \ldots, n]$. The problem consists in finding the minimum number $M$ of processes that guarantee the productive execution of $N$ strategic processes. Therefore, we merely need to require

$$
M^{*}=\min _{\Omega} \sum_{i=1}^{n} x_{i}
$$

The feasible region $\Omega$ is defined by $2 \mathrm{n}$ constraints. The first collection of $\mathrm{n}$ constraints is logical type. It permits to get $x_{i}=1$ when $s_{i}=1$, while $s_{i}=0$ implies $x_{i}=0$. Formally, the constraint depicts the logical implication:

- $\mathrm{s}_{\mathrm{i}}=1 \Rightarrow \mathrm{x}_{\mathrm{i}}=1$

- $\mathrm{S}_{\mathrm{i}}=0 \Rightarrow \mathrm{x}_{\mathrm{i}}=0$

The sector's outputs are not less than the required supply $\vec{s}$ if and only if

$$
\left\{\begin{array}{c}
x_{1} \geq s_{1} \\
x_{2} \geq s_{2} \\
\vdots \\
x_{n} \geq s_{n}
\end{array}\right.
$$

The $\mathrm{n}$ constraints can be written as a single matrix inequality $\vec{x} \cdot I \geq \vec{s}$ where $I$ is the identity matrix and $\cdot$ is the matrix product between the row $\vec{x}$ and the columns of $I$.

The second collection of $\mathrm{n}$ constraints is very important because it permits to guarantee the proper level of inputs for strategic sectors coming from other directly or indirectly joined sectors.

$$
\left\{\begin{array}{c}
\sum_{i=1}^{n} x_{i} \cdot a_{i 1} \geq x_{1} \cdot \sum_{i=1}^{n} a_{i 1} \\
\sum_{i=1}^{n} x_{i} \cdot a_{i 2} \geq x_{2} \cdot \sum_{i=1}^{n} a_{i 2} \\
\vdots \\
\sum_{i=1}^{n} x_{i} \cdot a_{i n} \geq x_{n} \cdot \sum_{i=1}^{n} a_{i n}
\end{array}\right.
$$

The $\mathrm{n}$ inequalities mean that if a $\mathrm{k}$-th process is strategic, that is $\mathrm{x}_{\mathrm{k}}=1$, then the right side of the inequality, $x_{k} \cdot \sum_{i=1}^{n} a_{i k}$, cannot be zero if $\sum_{i=1}^{n} a_{i k} \neq 0$. As $x_{k} \cdot \sum_{i=1}^{n} a_{i k} \neq 0$ represents the total input to the $\mathrm{k}$-th sector and since this supply is necessary for the $\mathrm{k}$ th sector to receive inputs for its own activities, the scalar product $\sum_{i=1}^{n} x_{i} \cdot a_{i k}$ 
determines which sectors are essential because they furnish inputs to process $\mathrm{k}$. Therefore, these sectors cannot take null value. Indicated with $b_{k}=\sum_{i=1}^{n} a_{i k}$ we have

$$
\tilde{A}=\left(\begin{array}{ccc}
a_{11}-b_{1} & \ldots & a_{1 n} \\
\vdots & \ddots & \vdots \\
a_{n 1} & \ldots & a_{n n-b_{n}}
\end{array}\right)
$$

and we can write $\vec{x} \cdot \tilde{A} \geq \overrightarrow{0}$ as expression of the last $\mathrm{n}$ inequalities.

The model can be formulated in a compact way like

$$
\begin{gathered}
\min _{\Omega} \sum_{i=1}^{n} x_{i} \\
\Omega:\left\{\begin{array}{c}
I \cdot \vec{x}^{t} \geq \vec{s} \\
\widetilde{A}^{t} \cdot \vec{x}^{t} \geq \overrightarrow{0} \\
x_{i} \in\{0 ; 1\}
\end{array}\right.
\end{gathered}
$$

It should be noted that if $N=0$ then $M^{*}=0$. In general, we expect $0<N \leq M^{*} \leq \mathrm{n}$. Secondly, if $N<M^{*}<n$ occurs it means that it is possible to lockdown n- $M^{*}$ sectors, but reducing the number of opened sectors to $N$ will probably cause a negative impact on the supply-chain as a result of an insufficient and inadequate level of inputs.

The dual problem is introduced in the case of the continuous relaxation of the problem (1): components of the variable $\vec{x}$ no longer take integer values (boolean to be exact), but they can assume continuous values in the interval $[0,1]$. The relaxed formulation related to the minimal lockdown problem is:

$$
\begin{aligned}
& M_{r}^{*}=\min _{\Omega} \sum_{i=1}^{n} x_{i} \\
& \Omega:\left\{\begin{array}{l}
I \cdot \vec{x}^{t} \geq \vec{s} \\
\vec{A}^{t} \cdot \vec{x}^{t} \geq \overrightarrow{0} \\
I \cdot \vec{x}^{t} \leq \overrightarrow{1} \\
\vec{x} \geq \overrightarrow{0}
\end{array}\right.
\end{aligned}
$$

The dual formulation of the primal problem $(* *)$, is obtained by writing the Lagrangian function $\mathrm{L}$ of the primal problem and connecting such function to minimax theorem of John von Neumann [9]:

$$
\max _{\vec{\lambda}_{1}, \vec{\lambda}_{2}, \vec{\lambda}_{3}} \min _{\vec{x}} L\left(\vec{x}, \vec{\lambda}_{1}, \vec{\lambda}_{2}, \vec{\lambda}_{3}\right)=\min _{\vec{x}} \max _{\vec{\lambda}_{1}, \vec{\lambda}_{2}, \vec{\lambda}_{3}} L\left(\vec{x}, \vec{\lambda}_{1}, \vec{\lambda}_{2}, \vec{\lambda}_{3}\right)
$$

The Lagrangian function to be considered is:

$$
L\left(\vec{x}, \vec{\lambda}_{1}, \vec{\lambda}_{2}, \vec{\lambda}_{3}\right)=<\overrightarrow{1}, \vec{x}^{t}>+<\vec{\lambda}_{1}, \vec{s}-I \cdot \vec{x}^{t}>+<\vec{\lambda}_{2}, \tilde{A}^{t} \cdot \vec{x}^{t}>-<\vec{\lambda}_{3}, \overrightarrow{1}-I \cdot \vec{x}^{t}>
$$


where notation $<\cdot,>>$ indicates the scalar product between two vectors; $\vec{\lambda}_{1}, \vec{\lambda}_{2}, \vec{\lambda}_{3}$ are Lagrange multipliers. Taking advantage of the linearity of the scalar product, remembering that $\langle\overrightarrow{\mathrm{v}}, A \cdot \overrightarrow{\mathrm{w}}\rangle=<A^{t} \cdot \overrightarrow{\mathrm{v}}, \overrightarrow{\mathrm{w}}>$ and putting in evidence variable $\vec{x}^{t}$, we get $L\left(\vec{x}, \vec{\lambda}_{1}, \vec{\lambda}_{2}, \vec{\lambda}_{3}\right)=<\vec{\lambda}_{1}, \vec{s}>-<\vec{\lambda}_{3}, \overrightarrow{1}>+<\overrightarrow{1}-I \cdot \vec{\lambda}_{1}-\tilde{A} \cdot \vec{\lambda}_{2}+I \cdot \vec{\lambda}_{3}, \vec{x}^{t}>$.

Minimizing $L\left(\vec{x}, \vec{\lambda}_{1}, \vec{\lambda}_{2}, \vec{\lambda}_{3}\right)$ with respect to variable $\vec{x} \geq \overrightarrow{0}$ is equivalent to consider the minimum of $\left\langle\overrightarrow{1}-I \cdot \vec{\lambda}_{1}-\tilde{A} \cdot \vec{\lambda}_{2}+I \cdot \vec{\lambda}_{3}, \vec{x}^{t}\right\rangle$. This minimum is finite and equal to 0 if and only if $\overrightarrow{1}-I \cdot \vec{\lambda}_{1}-\widetilde{A} \cdot \vec{\lambda}_{2}+I \cdot \vec{\lambda}_{3} \geq \overrightarrow{0}$. The dual formulation of (2) is expressed as

$$
\begin{array}{r}
\max _{\Omega^{\prime}}\left[\sum_{i=1}^{n} \lambda_{1 i} \cdot s_{i}-\sum_{i=1}^{n} \lambda_{3 i}\right] \\
\Omega^{\prime}:\left\{\begin{array}{l}
I \cdot \vec{\lambda}_{1}+\widetilde{A} \cdot \vec{\lambda}_{2}-I \cdot \vec{\lambda}_{3} \leq \overrightarrow{1} \\
\vec{\lambda}_{1} \geq \overrightarrow{0} \\
\vec{\lambda}_{2} \geq \overrightarrow{0} \\
\vec{\lambda}_{3} \geq \overrightarrow{0}
\end{array}\right.
\end{array}
$$

Thinking to Lagrange multipliers as shadow prices, they can be interpreted as relative value for closure or opening sector with regards to its contribution inside the economic system.

\section{Interpreting Lagrange multipliers like potential values of the sectors inside the} economic system: In order to take advantage of the Lagrange multiplier theorem it is necessary to formulate the feasible region $\Omega$ like a manifold $\mathrm{V}$ defined by means of a vector equation such as $\vec{F}(\vec{z})=\overrightarrow{0}$ where the variable $\vec{z}$ has $\mathrm{n}+2 \mathrm{~m}$ components. Introducing $\mathrm{m}$ surplus variables $\mathrm{u}_{\mathrm{j}}$ the $\mathrm{m}$ constraints in the form of inequality will be expressed as m equations $F_{j}(\vec{z})=0$ where $\mathrm{j}=1, \ldots, \mathrm{m}$. Considered that in the model (2)

$\vec{b}=(\underbrace{s_{1}, \ldots, s_{n}, \overbrace{0, \ldots, 0}^{n}, \overbrace{-1, \ldots,-1}^{n}}_{m=3 n})$

$\vec{u}=\left(u_{1}, \ldots, u_{n}, u_{n+1}, \ldots, u_{2 n}, u_{2 n+1}, \ldots, u_{3 n}\right)$ so that $m=3 n$

$\vec{z}=(\vec{y}, \vec{b})=\left(x_{1}, \ldots, x_{n}, u_{1}, \ldots, x_{3 n}, b_{1}, \ldots, b_{3 n}\right)$ so that $\mathrm{k}=\mathrm{n}+2 \mathrm{~m}=\mathrm{n}+2 \cdot(3 \mathrm{n})=7 \mathrm{n}$

then the model for optimal economic lockdown to contain epidemic can be interpreted like 


$$
\min _{\mathrm{V}} \sum_{i=1}^{k} c_{i} \cdot z_{i}=\min _{\mathrm{V}}\left[\sum_{i=1}^{n} x_{i}+\sum_{i=n+1}^{2 n} 0 \cdot u_{i}+\sum_{i=2 n+1}^{3 n} 0 \cdot b_{i}\right]=\min _{\mathrm{V}} \sum_{i=1}^{n} x_{i}
$$

The linear manifold $\mathrm{V}$ is defined as a system of $3 \mathrm{n}$ equations in $\mathrm{n}$ variables,

for $1 \leq \mathrm{j} \leq \mathrm{n}$ we have

$F_{j}\left(x_{1} \ldots, x_{n}, u_{1}, \ldots, u_{3 n}, b_{1}, \ldots, b_{3 n}\right)=g_{j}\left(x_{1} \ldots, x_{n}\right)-u_{j}-b_{j}=x_{j}-u_{j}-s_{j}=0$

for $\mathrm{n}+1 \leq \mathrm{j} \leq 2 \mathrm{n}$ we have

$F_{j}(\vec{x}, \vec{u}, \vec{b})=g_{j}\left(x_{1} \ldots, x_{n}\right)-u_{j}-b_{j}=<\tilde{a}^{j}, \vec{x}>-u_{j}-0=0$,

for $2 \mathrm{n}+1 \leq \mathrm{j} \leq 3 \mathrm{n}$ we have

$F_{j}(\vec{x}, \vec{u}, \vec{b})=g_{j}\left(x_{1} \ldots, x_{n}\right)-u_{j}-b_{j}=-x_{k}-u_{j}+1=0$ with $1 \leq \mathrm{k} \leq \mathrm{n}$

The standard form of the model (2) is expressed as

$$
B \cdot \vec{y}^{t}=\vec{b}^{t}
$$

with B block matrix $3 n \times 3 n$ whose rank is equal to $3 n$,

$$
\left(\begin{array}{ccc}
I & -I & 0 \\
\tilde{A}^{t} & 0 & 0 \\
-I & 0 & -I
\end{array}\right) \cdot\left(\begin{array}{c}
x_{1} \\
\vdots \\
u_{3 n}
\end{array}\right)=\left(\begin{array}{c}
b_{1} \\
\vdots \\
b_{3 n}
\end{array}\right)
$$

$I$ is the identity matrix $\mathrm{n} \mathrm{n}$.

The Lagrange multipliers theorem states that if $\Phi$ attains a constrained local minima in $\vec{z}_{0}$ then there exist $m$ real number $\lambda_{1}, \ldots, \lambda_{\mathrm{m}}$ such that $\nabla \Phi\left(\vec{z}_{0}\right)=\sum_{j=1}^{m} \lambda_{j} \cdot \nabla F_{j}\left(\vec{z}_{0}\right)$.

Formula is written in full as

$$
\nabla \Phi \equiv \sum_{i=1}^{k} \frac{\partial \Phi\left(\vec{z}_{0}\right)}{\partial z_{i}} \cdot h_{i}=\sum_{j=1}^{m} \lambda_{j} \cdot \sum_{i=1}^{n+2 m} \frac{\partial F_{j}\left(\vec{y}_{0}, \vec{b}_{0}\right)}{\partial z_{i}} \cdot h_{i}
$$

in a neighbourhood of $\vec{z}_{0}$. For sufficiently small variations of $\vec{h}$ variation, the variation of the objective function $\Phi$ can be approximated by its gradient: so, the approximation $\Delta \Phi \sim \nabla \Phi$ holds. The same holds for the functions $\mathrm{F}_{\mathrm{j}}: \Delta \mathrm{F}_{\mathrm{j}} \sim \nabla \mathrm{F}_{\mathrm{j}}$. 
As a result, we deduce that

$$
\Delta \Phi \sim \sum_{j=1}^{m} \lambda_{j} \cdot \sum_{i=1}^{n+2 m} \Delta F_{j}
$$

If $\Phi$ and $F_{j}$ are linear transformations, it is true that $\mathrm{d} \Phi=\Phi$ and $\mathrm{dF}_{\mathrm{j}}=\mathrm{F}_{\mathrm{j}}$. Therefore, the equality sign holds

$$
\Delta \Phi=\sum_{j=1}^{m} \lambda_{j} \cdot \sum_{i=1}^{n+2 m} \Delta F_{j}
$$

We can observe that the linearity of gradient implies

$\nabla F_{j}=\nabla\left(g_{j}(\vec{x})+u_{j}-b_{j}\right)=\nabla g_{j}(\vec{x})+\frac{\partial u}{\partial u_{j}}-\frac{\partial b}{\partial b_{j}}=\nabla g_{j}(\vec{x})+1-1=\nabla g_{j}(\vec{x})$

so, it is true that in a neighbourhood of $\vec{z}_{0}=\left(\vec{x}_{0}, \vec{u}_{0}, \vec{b}_{0}\right)$ we have

$$
\Delta \Phi=\sum_{j=1}^{m} \lambda_{j} \cdot \sum_{i=1}^{n+2 m} \Delta g_{j}
$$

In $1^{\text {st }}$ group of equations $1 \leq \mathrm{j} \leq \mathrm{n}$ it is $\Delta g_{j}=h_{j}$; in $2^{\text {nd }}$ group of equations $\mathrm{n}+1 \leq \mathrm{j} \leq 2 \mathrm{n}$ it is $\Delta g_{j}=<\tilde{a}^{j}, \vec{h}>$; in $3^{\text {rd }}$ group of equations $2 \mathrm{n}+1 \leq \mathrm{j} \leq 3 \mathrm{n}$ it is $\Delta g_{j}=-h_{j}$.

Using formula (5) the variation $\Delta \Phi$ is given by

$$
\lambda_{1} \cdot h_{1}+\cdots+\lambda_{n} \cdot h_{n}+\lambda_{n+1} \cdot \widetilde{\boldsymbol{a}}^{1} \cdot \overrightarrow{\boldsymbol{h}}+\cdots+\lambda_{2 n} \cdot \widetilde{\boldsymbol{a}}^{n} \cdot \overrightarrow{\boldsymbol{h}}-\lambda_{2 n+1} \cdot h_{1}-\cdots-\lambda_{3 n} \cdot h_{n}
$$

If same sectors are grouped, we get

$$
\Delta \Phi=\underbrace{\left(\lambda_{1} \cdot h_{1}+\lambda_{n+1} \cdot \widetilde{\boldsymbol{a}}^{1} \cdot \overrightarrow{\boldsymbol{h}}-\lambda_{2 n+1} \cdot h_{1}\right)}_{1 \text { st sector }}+\cdots+\underbrace{\left(\lambda_{n} \cdot h_{n}+\lambda_{2 n} \cdot \widetilde{\boldsymbol{a}}^{n} \cdot \overrightarrow{\boldsymbol{h}}-\lambda_{3 n} \cdot h_{n}\right)}_{n \text {-th sector }}
$$

For convenience of notation, let's indicate the expression in round brackets of a generic sector $1 \leq \mathrm{k} \leq \mathrm{n}$ like $\Lambda_{k}:=\lambda_{k} \cdot h_{k}+\lambda_{n+k} \cdot \widetilde{\boldsymbol{a}}^{k} \cdot \overrightarrow{\boldsymbol{h}}-\lambda_{2 n+k} \cdot h_{k}$. We can write

$$
\Delta \Phi=\sum_{j=1}^{n} \Lambda_{j}
$$

Now, it is possible to assess the sensitivity of optimal value $\Phi\left(\vec{z}_{0}\right)$ to variations in the values $s_{1}, \ldots, s_{n}$ referred to $\vec{b}$. The Lagrange multiplier can be interpreted as a potential value, an opportunity to represent the benefit that would be obtained in giving up an economic sector that was initially qualified as strategic. The opportunity to have one 
less strategic sector lies in the fact that the resulting advantage is measured by the decrease in the objective function, that is, by the decrease in the count of the sectors that must remain open.

As said applies to a "perturbation" on a single sector which is left closed as a strategic one; what can be said about the essential sectors? The essential sectors are all those sectors considered not strategic by the legislator, but for which the optimal solution of the lockdown provides that they are left open. We ask if the comparison the Lagrange multipliers' values could lead to establish a "ranking", a preferential ordering which assigns priority to essential sectors so that they can gradually reopen after the containment of the epidemic. The resulting benefit would come from the number of supply flows (inputs) restored. Opening essential sectors helps the whole economic system because supply chains can operate without more bottlenecks in inputs.

4. What sectors should be reopened before: The essential sectors are all those sectors considered not strategic by the legislator, but for which the optimal solution of the lockdown provides that they are left open: the $\mathrm{i}$-th sector $1 \leq \mathrm{i} \leq \mathrm{n}$ is essential if $\mathrm{s}_{\mathrm{i}}=0$ and at the extremum point results $\mathrm{x}_{\mathrm{i}}=1$. The Lagrange multiplier theorem determines the impact on the objective function following "small" variations in extrema points, we want to establish which of the $\mathrm{k}$ essential sectors have the greatest effect in the variation of the objective function. Given the i-th sector as essential, by definition it means that $\mathrm{s}_{0 \mathrm{i}}=0$ e $\mathrm{x}_{0 \mathrm{i}}=1$. Following the $\delta$ perturbation which brings the $\mathrm{i}$-th sector from being defined as essential to being closed $x_{1 i}=0, \mathrm{~s}_{1 \mathrm{i}}=\mathrm{s}_{\mathrm{oi}}+\delta=1$, the variation of the objective function is $\Delta \Phi=-1$. Chosen $\delta=1$, from $s_{0 i}=0$ we get $s_{1 i}=1$

$$
\left(x_{01}, \ldots, x_{0 i}=1, \ldots, x_{0 n}\right) \mapsto\left(x_{01}, \ldots, x_{1 i}=0, \ldots, x_{0 n}\right), \overrightarrow{\boldsymbol{h}}=(0, \ldots, 0, \underbrace{-1}_{i}, 0, \ldots, 0)
$$

In the $1^{\text {st }}$ group of equations $1 \leq \mathrm{i} \leq \mathrm{n}$ it turns out $\Delta g_{i}=h_{i}=x_{1 i}-x_{0 i}=0-1=-1$. Since $\mathrm{s}_{0 \mathrm{i}}=0$ and $\mathrm{x}_{0 \mathrm{i}}=1$, the corresponding surplus variable differs from zero, $\mathrm{u}_{\mathrm{oi}} \neq 0$, the complementary slackness theorem implies that the corresponding Lagrange multiplier is $\lambda_{i}=0$. In the $2^{\text {nd }}$ group of equations $\mathrm{n}+1 \leq \mathrm{i} \leq 2 \mathrm{n}$ it turns out $\Delta g_{i}=<\tilde{a}^{i}, \vec{h}>$ $=a_{i i}-\sum_{k=1}^{n} a_{k i}<0$, therefore the corresponding surplus variable is not zero and the complementary slackness theorem implies $\lambda_{n+i}=0$.

In the $3^{\text {rd }}$ group of equations $2 \mathrm{n}+1 \leq \mathrm{i} \leq 3 \mathrm{n}$ it is $\Delta g_{i}=-h_{i}=1$, since the corresponding constraint is binding $-x_{0 i}-u_{0 i}+1=0$, the complementary slackness theorem 
implies $\lambda_{2 n+i}>0$. For all others constraints $\mathrm{j} \neq \mathrm{i} 1 \leq \mathrm{j} \leq \mathrm{n}$ it is $\Delta g_{i}=0$ in $1^{\text {st }}$ and $3^{\text {rd }}$ group, while in the $2^{\text {nd }}$ group is

$$
\Delta g_{j}=<\tilde{a}^{j}, \vec{h}>=\left\{\begin{array}{lll}
0 & \text { se } & a_{i j}=0 \\
1 & \text { se } & a_{i j}=1
\end{array}\right.
$$

it is deduced that

$$
\Delta \Phi=\sum_{j=1}^{n} \Lambda_{j}=\Lambda_{i}+\sum_{\substack{j=1 \\ j \neq i}}^{n-1} \lambda_{n+j} \cdot a_{i j}=-1
$$

namely

$$
\Lambda_{i}=\lambda_{2 n+i}=-1-\sum_{\substack{j=1 \\ j \neq i}}^{n-1} \lambda_{n+j} \cdot a_{i j}
$$

Since $\lambda_{2 n+i}>0$, it necessary occurs $\sum_{\substack{j=1 \\ j \neq i}}^{n-1} \lambda_{n+j} \cdot a_{i j}<-1$

Suppose that the solution to problem (2) shows k sectors as essential. The solution of the dual problem (3) can therefore be adopted in order to define priority to reopen the essential sectors in the phase after the containment of the epidemic. $\Delta \Phi$ is maximized if we choose the largest value of $\Lambda_{i}$. Let's introduce the symbol $>$ as binary relationship called precedence: if sector $i$ and sector $j$ are two essential sectors, the sector $i$ must be opened before the opening of sector $i$ and it is written

$$
\text { sector } i_{[1]}>\text { sector } j_{[2]}
$$

if

$$
\Lambda_{i}>\Lambda_{j}
$$

Square brackets will be used to denote position in sectors' sequence. The symbol [1] means the $\mathrm{i}$-th sector is in the first position in sequence.

Among k essential sectors, we choose Tra questi k settori the sector with the largest $\Lambda_{i}$ is chosen as the sector in [1] position, in [2] position it will be necessary to choose the sector which among the remaining ones has the highest $\Lambda_{1}, \ldots, \Lambda_{k-1}$. Proceeding in this way, we build a sequence of sectors in which the sector in [i] position has the highest among remaining ones. Finally, the sequence

$$
\text { sector } i_{[1]}>\text { sector } j_{[2]}>\cdots>\text { sector } m_{[k]}
$$

represents the order to reopen sectors which Decree has closed and model has been considering as essential. 
5. The case of Italian economy, $\mathbf{n}=63$ : On national basis, Istat makes available Supply and Use Tables for 2015-2016. Economic activities are classified in accordance with Nace Rev. 2, while products are classified in accordance with Cpa 2008. The two classifications are perfectly aligned with each other. The optimal lockdown model has been run with adjacency matrix got from the Istat Supply Table: "SUPPLY_63B.xls". The level of detail of this Table is 63 sectors of economic activities and 63 ensembles of products. At first the correspondence was found between the ATECO codes of the "Close Italy" Decree and the Nace rev.2 classification of the economic sectors. The sectors considered strategic by the Decree amount to 47 out of a total of 63 sectors monitored by Istat.

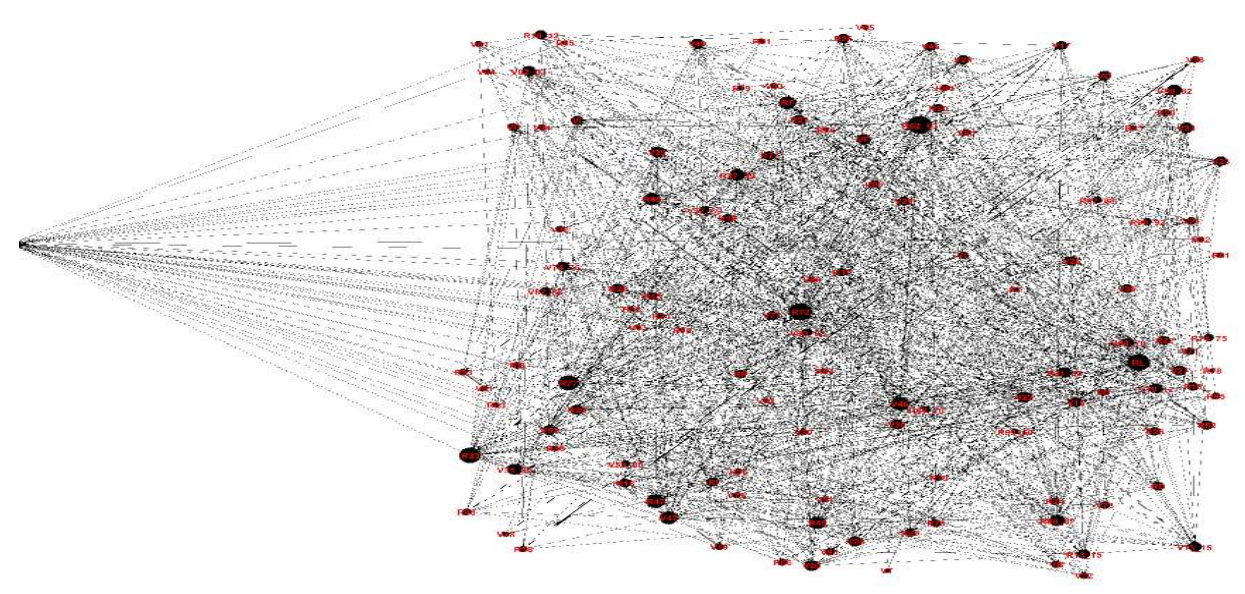

Figure 1. Existing interdependences in Italian economy

The supply demand vector $\vec{s}$ has been introduced like stated in "Close Italy" Decree. The solution of the optimal lockdown model specifies to close only one sector

- V78 - Research, selection, personnel activities.

This sector is related to the product R78 - Job services.

Model keeps open all remaining 62 sectors, so that 15 sectors should be considered as essential for Italian economy operating with 47 strategic sectors.

6. The gradual process to reopen sector by sector: The model leads to consider the sectors associated with 15 goods / services as essential: although the "Close Italy" Decree rightly considered them to be closed, the related activities are necessary for the economic system. The 15 goods / services indicated as essential by the model are shown 
in table 1 . These 15 sectors will have a significant impact on an economic system in which 47 interdependencies have been suspended.

Table 1: Ranking by priority for reopening of essential sectors

\begin{tabular}{|c|c|c|c|c|}
\hline Priority & Sector & $\Lambda$ & input & output \\
\hline 1 & Real estate services & 2.51 & 16 & 58 \\
\hline 2 & $\begin{array}{l}\text { Retail services, except of motor vehicles and } \\
\text { motorcycles }\end{array}$ & 1.81 & 41 & 45 \\
\hline 3 & Rental and leasing services & 1.54 & 21 & 50 \\
\hline 4 & $\begin{array}{l}\text { Construction works and civil construction } \\
\text { works }\end{array}$ & 1.02 & 30 & 39 \\
\hline 5 & Other means of transportation & 0.81 & 25 & 20 \\
\hline 6 & Metal products, excluding machines and plants & 0.49 & 31 & 24 \\
\hline 7 & Advertising and market research services & 0.28 & 16 & 17 \\
\hline 8 & Accommodation and restaurant services & 0.23 & 22 & 22 \\
\hline 9 & Other personal services & 0.09 & 21 & 11 \\
\hline 10 & Motor vehicles, trailers and semitrailers & 0.08 & 22 & 14 \\
\hline 11 & Metals & 0 & 24 & 15 \\
\hline 11 & $\begin{array}{l}\text { Creative, artistic and entertainment services; } \\
\text { library, archives, museum and other cultural } \\
\text { services; gambling services }\end{array}$ & 0 & 15 & 12 \\
\hline 11 & Sports and entertainment services & 0 & 15 & 11 \\
\hline 11 & Publishing services & 0 & 11 & 10 \\
\hline 11 & $\begin{array}{l}\text { Travel agency, tour operator and other booking } \\
\text { services and related services }\end{array}$ & 0 & 8 & 9 \\
\hline
\end{tabular}

The column "input" shows the number of sectors as suppliers, while the column "output" shows the number of served sectors. It should be noted that connection among sectors is measured by $\Lambda$ : large $\Lambda$ means large amounts of served sectors (output). 
7. Conclusions: The mathematical model for optimal lockdown allows to establish the feasibility of interconnected sectors' closure within the Italian economy. On the other hand, the model can be of support in assigning priorities to sectors which can be gradually reopened as the spread of the virus decreases. As observed, a large Lagrange multiplier corresponds to a sector which is relevant in terms of its interdependencies. The model recommends to opening before the closed sectors with large Lagrange multipliers.

Lastly, in order to reduce the risk of contagion every sector could be distinguished with the number of workers directly involved in the operations of the sectors, so that the number of workers relieved from work could be minimized.

8. Acknowledgements: Many thanks to Professor Michelangelo Puliga for the encouragement and insightful discussions we had at the beginning of the work.

\section{References:}

[1] Harvey M. Wagner, "A Linear Programming Solution to Dynamic Leontief type Models", RAND Co., 1957

[2] Yacov Y. Haimes, Barry M. Horowitz, James H. Lambert, Joost R. Santos, Chenyang Lian, Kenneth G. Crowther, "Inoperability Input-Output Model for Interdependent Infrastructure Sectors. I: Theory and Methodology" in Journal of Infrastructure Systems Volume 11 Issue 2, 2005

[3] Yacov Y. Haimes, Barry M. Horowitz, James H. Lambert, Joost R. Santos, Chenyang Lian, Kenneth G. Crowther, "Inoperability Input-Output Model for Interdependent Infrastructure Sectors. II: Case Studies" in Journal of Infrastructure Systems Volume 11 Issue 2, 2005

[4] Erik Dietzenbacher, Ronald E. Miller, "Reflections on the Inoperability InputOutput Model”, Economic Systems Research, Volume 27 Issue 4, 2015

[5] Shaowen Luo, Kwok Ping Tsang, "China and World Output Impact of the Hubei Lockdown During the Coronavirus Outbreak", Forthcoming, Contemporary Economic Policy, 2020

[6] Leontief W., "Input-Output Economics" 2nd ed., Oxford University Press, 1986 
[7] Miller, R. E., Blair, P.D. "Input-Output-Analysis. Foundations and Extensions" 2nd ed., Cambridge University Press, 2009

[8] Thijs, ten Raa, "Handbook of Input-Output Analysis", Edward Elgar, 2017

[9] B. Güler, "Foundations of Optimization," Springer-Verlag, 2010

[10] The Input-Output Tables published on 05.02 .2020 by Istat and they are available at the following link: https://www.istat.it/it/archivio/238228 